\title{
Genetic Predictors of Increase in Suicidal Ideation During Antidepressant Treatment in the GENDEP Project
}

\begin{abstract}
Nader Perroud*,1,12, Katherine J Aitchison',12, Rudolf Uher', Rebecca Smith', Patricia Huezo-Diaz', Andrej Marusic ${ }^{2}$, Wolfgang Maier ${ }^{3}$, Ole Mors ${ }^{4}$, Anna Placentino ${ }^{5}$, Neven Henigsberg ${ }^{6}$, Marcella Rietschel ${ }^{7}$, Joanna Hauser ${ }^{8}$, Daniel Souery ${ }^{9}$, Pawel Kapelski $^{8}$, Cristian Bonvicini $^{5}$, Astrid Zobel ${ }^{3}$, Lisbeth Jorgensen ${ }^{10}$, Ana Petrovic ${ }^{2}$, Petra Kalember', Thomas G Schulze 7 , Bhanu Gupta', Joanna Gray', Cathryn M Lewis', I', Anne E Farmer', Peter McGuffin' and lan Craig'
\end{abstract}

'MRC Social Genetic and Developmental Psychiatry Center, Institute of Psychiatry, King's College Lodon, London, UK; Institute of Public Health, Trubarjeva 2, Ljubljana, Slovenia; ${ }^{3}$ Department of Psychiatry, University of Bonn, Sigmund-Freud-Str 25, Bonn, Germany; ${ }^{4}$ Centre for Psychiatric Research, Aarhus University Hospital, Skovagenvej 2, Risskov, Denmark; ${ }^{5}$ Biological Psychiatry Unit and Dual Diagnosis ward IRCCS, Centro San Giovanni di Dio, FBF, Brescia, Italy; ${ }^{6}$ Department of Neuropharmacology and Behavioural Pharmacology, Croatian Institute for Brain Research, Medical School, University of Zagreb, Salata 12, Zagreb, Croatia; 'Division of Genetic Epidemiology in Psychiatry, Central Institute of Mental Health, Mannheim, Germany; ${ }^{8}$ Department of Psychiatry, Laboratory of Psychiatric Genetics, Poznan University of Medical Sciences, Poznan, Poland; ${ }^{9}$ Department of Psychiatry, Laboratoire de Psychologie Médicale, Université Libre de Bruxelles and Psy Pluriel—Centre Européen de Psychologie Médicale, Brussels, Belgium; ${ }^{10}$ Mood Disorders Research Unit, Aarhus University Hospital, Risskov, Risskov, Denmark; "'King's College London, Department of Medical and Molecular Genetics, London, UK

\begin{abstract}
The aim of this study was to investigate genetic predictors of an increase in suicidal ideation during treatment with a selective serotonin reuptake inhibitor or a tricyclic antidepressant. A total of 796 adult patients with major depressive disorder who were treated with a flexible dosage of escitalopram or nortriptyline in Genome-based Therapeutic Drugs for Depression (GENDEP) were included in the sample and provided data on suicidal ideation. Nine candidate genes involved in neurotrophic, serotonergic, and noradrenergic pathways were selected based on previous association studies with suicidal ideation or behavior. Using a logistic regression model, 123 polymorphisms in these genes were compared between subjects with an increase in suicidal ideation and those without any increase in suicidal ideation. Polymorphisms in BDNF, the gene encoding the brain-derived neurotrophic factor, were significantly associated with an increase in suicidal ideation. The strongest association was observed for rs962369 in BDNF $(p=0.0015)$. Moreover, a significant interaction was found between variants in BDNF and NTRK2, the gene encoding the BNDF receptor $(p=0.0003)$. Among men taking nortriptyline, suicidality was also associated with rs I I 195419 SNP in the alpha 2 A-adrenergic receptor gene $(A D R A 2 A)(p=0.007)$. The associations observed with polymorphisms in BDNF suggest the involvement of the neurotrophic system in vulnerability to suicidality. Epistasis between BDNF and NTRK2 suggests that genetic variations in the two genes are involved in the same causal mechanisms leading to suicidality during antidepressant treatment. Among men, genetic variation in noradrenergic signaling may interact with norepinephrine reuptake-inhibiting antidepressants, thereby contributing to suicidality.

Neuropsychopharmacology (2009) 34, 25 I7-2528; doi:I 0. I038/npp.2009.8I; published online 29 July 2009
\end{abstract}

Keywords: suicidal ideation; antidepressive agents; BDNF; norepinephrine; serotonin; ADRA2A

\section{INTRODUCTION}

Emergence or worsening of suicidal ideation are among the most serious adverse events on antidepressant treatment. Reports of these risks have led regulatory authorities to

*Correspondence: Dr N Perroud, Institute of Psychiatry at King's College London, MRC SGDP Centre, Internal PO80, 16 De Crespigny Park, Denmark Hill, SE5 8AF, London, United Kingdom, Tel: + 44207 848 089।, Fax: + 44207848 0866, E-mails: nader.perroud@iop.kcl. ac.uk or nader.perroud@hcuge.ch

${ }^{12}$ These two authors contributed equally to this paper.

Received 7 April 2009; revised 21 May 2009; accepted 22 June 2009 issue warnings to clinicians (US Food and Drug Administration, 2006).

Twin and adoption studies suggest a genetic contribution to suicidal behavior (SB) (Brent and Mann, 2005; McGuffin et al, 2001). As low central nervous system serotonin (5-HT) turnover is related to SB (Mann, 2003), serotonin-related genes have been the focus of several association studies (Courtet et al, 2005). Meta-analyses have confirmed the association of SB with variants in the serotonin transporter gene (5-HTT, or SLC6A4) ( $\mathrm{Li}$ and $\mathrm{He}, 2007$ ) and the tryptophan hydroxylase 1 gene (TPH1) (Bellivier et al, 2004). The genes encoding brain-derived neurotrophic factor $(B D N F)$ and its receptor, the neurotrophic tyrosine 
kinase receptor type 2 (NTRK2) are also promising candidates as they are involved in the regulation and growth of 5-HT neurons (Perroud et al, 2008). Noradrenergic pathways and especially the alpha $\mathrm{eA}_{\mathrm{A}}$-adrenergic receptor (encoded by $A D R A 2 A$ ) have also been implicated in suicide (Escriba et al, 2004; Sequeira et al, 2004).

Although the genetics of spontaneously occurring SB has been researched for decades, the genetic determinants of suicidality during antidepressant treatment are only beginning to be uncovered. Recent pharmacogenetic investigations suggest that emergence of suicidal ideation during antidepressant treatment might be genetically driven (Laje et al, 2007; Perlis et al, 2007). It has also been hypothesized that acute reduction in central BDNF expression and dysfunction in the NTRK2 pathway are involved in treatment-related suicidal ideation (Tsai, 2005). We have tested this hypothesis in the Genome-based Therapeutic Drugs for Depression (GENDEP) clinical sample, a multicenter European pharmacogenetic study, which has examined the efficacy and adverse effect profiles of a serotonergic and a noradrenergic antidepressant in a relatively large cohort of depressed subjects. Based on the available previous evidence, we selected nine candidate genes involved in neurotrophic, serotonergic, and noradrenergic pathways and compared genetic variants in these genes between subjects with and without an increase in suicidal ideation over the 12 weeks of treatment with the study antidepressants.

We have previously shown that increases in suicidal ideation in GENDEP were most common among men taking nortriptyline (Perroud et al, submitted). Therefore, we have also tested the hypothesis that specific noradrenergic and serotonergic genes differentially affect suicidality during treatment with nortriptyline and escitalopram, respectively. For this purpose, we selected from the candidate genes genotyped in GENDEP those with previous data implicating them in the mechanism of action of the study drugs (escitalopram or nortriptyline) as follows: the serotonin receptors 1A (HTR1A) and 2A (HTR2A), TPH1, TPH2, and the serotonin transporter (SLC6A4) genes were investigated for association with suicidal ideation during treatment with escitalopram; the ADRA2A, and the norepinephrine transporter (SLC6A2), were analyzed for nortriptyline; and $B D N F$ and NTRK2 for both drugs.

\section{MATERIALS AND METHODS}

The sample consisted of 811 subjects with major depressive disorder from GENDEP, a part-randomized multi-center pharmacogenomic study (http://gendep.iop.kcl.ac.uk/ results.php). Fifteen subjects who had missing data on all three suicidality items at baseline were excluded. Therefore, 796 subjects (254 'cases' and 542 'controls,' Table 1) were available for this analysis. The sample has been described in detail elsewhere (Perroud et al, submitted; Uher et al, 2009b). In brief, participants were included if they met criteria for a major depressive episode of at least moderate severity, as defined by the DSM-IV and/or ICD-10 criteria, established using the Schedules for Clinical Assessment in Neuropsychiatry (SCAN Version 2.1). Two antidepressants representing the two most common mechanisms of antidepressant action were chosen: (1) escitalopram, a selective inhibitor of the serotonin transporter with no effect on norepinephrine reuptake (Sanchez et al, 2003) and (2) nortriptyline, a tricyclic antidepressant with a hundred times higher affinity for the norepinephrine transporter than for the serotonin transporter (Sanchez and Hyttel, 1999). Nortriptyline was selected in preference to the even more selective reboxetine, as reboxetine has a less robust

Table I Socio-demographic Characteristics of 236 Depressed Individuals With an Increase in Suicidal Ideation ('cases' for the purpose of this analysis) and 49I Depressed 'Controls' (ie, Without an Increase in Suicidal Ideation)

Increase of suicidal ideation during the 12 weeks of follow-up

\begin{tabular}{ccccccc}
\hline & No & & \multicolumn{2}{c}{ Yes } & & \\
\cline { 1 - 3 } \cline { 5 - 6 } Mean & SD & & Mean & SD & Stat & P-value \\
\hline 42.2 & 11.7 & 42.8 & 11.7 & 0.71 & 0.47 \\
0.89 & 0.6 & 1.01 & 0.58 & 2.43 & 0.02 \\
33.04 & 9.51 & 32 & 10.81 & 1.33 & 0.18 \\
1.75 & 0.59 & 1.88 & 0.73 & 2.73 & 0.007 \\
12.2 & 3.08 & 11.9 & 3.08 & 0.77 & 0.44
\end{tabular}

\begin{tabular}{|c|c|c|c|c|c|c|}
\hline & Number & Percentage & Number & Percentage & & \\
\hline Gender & 303 & 61.7 & 160 & 67.8 & 2.55 & 0.11 \\
\hline History of suicide attempts & 50 & 10.2 & 64 & 27.1 & 34.6 & $p<0.000$ I \\
\hline Married & 245 & 49.9 & 109 & 46.2 & 0.88 & 0.348 \\
\hline Working & 248 & 50.5 & 133 & 56.4 & 2.18 & 0.139 \\
\hline Drop out & 124 & 25.3 & 37 & 15.7 & 8.48 & 0.004 \\
\hline
\end{tabular}


efficacy record (Cipriani et al, 2009). Patients with no contraindications were randomly allocated to receive flexible dosage nortriptyline (50-150 mg daily) or escitalopram (10-30 mg daily) for 12 weeks, within a defined range (Uher et al, 2008; Uher et al, 2009b). Patients with contraindications for one of the drugs were allocated nonrandomly to the other antidepressant. Participants who could not tolerate the initially allocated medication or who did not experience sufficient improvement despite adequate dosage for 8 weeks were offered a change to the other medication. Participants who changed medication were then followed up using the same protocol as for the first antidepressant. All subjects were of European ethnicity, between 18 and 72 years of age. The exclusion criteria were: a first-degree relative with bipolar affective disorder or schizophrenia, a history of hypomanic or manic episode, mood incongruent psychotic symptoms, primary substance misuse or primary organic disease, current treatment with an antipsychotic or a mood stabilizer, and pregnancy or lactation.

The study protocol was approved by the research ethics committee of each center, and written informed consent was obtained. GENDEP is registered under the following references: EudraCT No. 2004-001723-38 (http://eudract. emea.europa.eu); current controlled trials ISRCTN03693000 (http://www.controlled-trials.com).

\section{Suicidal Ideations}

Suicidal ideation was assessed using the third item of the 17-item Hamilton Rating Scale for Depression (HDRS-17), the ninth item of the self-report 21-item Beck Depression Inventory (BDI) and the tenth item of the clinician-rated 10-item Montgomery-Asberg Depression Rating Scale (MADRS). A composite score was calculated using item response theory (IRT), which allowed the use of all available data and provided us with unbiased estimates in the presence of missing values (Perroud et al, 2009; Uher et al, 2008).

Increase in suicidal ideation was defined as any increase of at least $0.5 \mathrm{SD}$ on the standardized IRT Scale during the 12 weeks of follow-up, and reaching a level of at least $1 \mathrm{SD}$ above the lowest possible score. This definition corresponded to a score of 1 or more on BDI and HDRS-17, and 2 or more on MADRS, and captured all individuals scoring on at least two of the three scales.

A previous analysis of this dataset showed that individuals with treatment-emergent suicidal ideation and those with treatment-worsening suicidal ideation shared similar risk factors (Perroud et al, 2009). We therefore designated individuals with either emergence or worsening of suicidal ideation at any time point in the 12-week follow-up period (254) as 'cases' and those without any increase of suicidal ideation (542) as 'controls'.

\section{Genotyping}

A sample of up to $6 \mathrm{ml}$ of venous blood was collected in EDTA and frozen. DNA was extracted using a standard procedure (Freeman et al, 2003). The length polymorphism in the promoter of the serotonin transporter gene (5-HTTLPR), and the putatively functional single nucleotide polymorphism rs25531 (A>G) located within the 5HTTLPR were genotyped using a two-stage method as described elsewhere (Huezo-Diaz et al, 2008). The $-1040(\mathrm{GT}) \mathrm{n}$ dinucleotide repeat within the BDNF-linked complex polymorphic region (BDNF-LCPR) was amplified using fluorescent forward primers and products were resolved on an Applied Biosystems 3130 sequencer (ABI3130). As the alleles displayed a bimodall distribution, $-1040(\mathrm{GT}) \mathrm{n}$ alleles were collapsed into two categories (S and $\mathrm{L}$ ) according to Vincze et al (Vincze et al, 2008). In addition, single nucleotide polymorphisms (SNPs) were selected to tag DNA sequence variation within the nine candidate genes using the SNPTagger program and HAPMAP data on the CEPH CEU population with European ancestry; CEPH NCBI Build 35/UCSC hg17/May 2004 coordinates (de Bakker et al, 2005). Criteria for SNP selection included a minor allele frequency of $5 \%$ in the CEU population and a pairwise linkage disequilibrium measure of $r^{2}>=0.8$. The SNPs were genotyped using the SNPlex method. This included oligonucleotide ligation and polymerase chain reaction (PCR) technology for amplification of the products of ligation and allelic discrimination. The assay products were run on the ABI3130 and data were exported and analyzed using GeneMapper software. Eleven samples were genotyped as blind duplicates for all SNPlex genotypes and the agreement was $100 \%$ for all markers.

\section{Statistical Analysis}

Logistic regression was used to evaluate the associations between genetic polymorphisms and increase in suicidal ideation. An additive genetic model was assumed. All analyses were adjusted for age, gender, and center of recruitment.

As we have previously shown gender and drug effects on increasing suicidal ideation in this sample, we investigated the gene and gender drug by gene interactions. For the drug by gene interaction, we first investigated the effect of polymorphisms for each drug separately in PLINK (Purcell et al, 2007) by taking individuals during the first and second course of medication (ie, before and/or after a change or switch in study medication). The associations were adjusted for switching status. As stated in the introduction, for each drug, only the genes with previous evidence of implication in the mechanism of action of the drugs were analyzed: HTR1A, HTR2A, TPH1, TPH2, and SLC6A4 genes for association with escitalopram; ADRA2A, and SLC6A2 for nortriptyline; and both BDNF and NTRK2 for the two drugs.

Second, to check that the detected associations were not due to confounding by specific individual or center-ofrecruitment effects, significant SNPs were reanalyzed using a generalized linear latent and mixed model (GLLAMM) in STATA as previously described (Perroud et al, 2009). Using this method, we were able to include all individuals, that is, those who switched and those who did not switch medication, by relaxing the assumption of conditional independence in the responses of the same person and for the same center of recruitment by including a subjectspecific random intercept nested in a center-specific random intercept (Rabe-Hesketh and Skrondal, 2005). To ascertain the specificity of any associations to suicidality as opposed to general treatment response, we performed a sensitivity analysis with the magnitude of change in the 
previously described 'observed mood' dimension as a covariate (Uher et al, 2008). The 'observed mood' dimension is derived from items assessing mood, anhedonia activity, and anxiety, but does not contain any information from the suicidality items and thus does not overlap with suicidal ideation score. Moreover, to confirm that the detected associations were not because of confounding by history of suicide attempts, history of suicide attempts was also entered as a covariate in this sensitivity analysis.

As the US Food Drug Administration (FDA) (US Food and Drug Administration, 2006) report states that treatment-emergent suicidality is more common in individuals aged under 25 years of age, and that antidepressants have a strong protective effect against suicidality in subjects aged 65 and older, the genetic association analyses were also reported separately for individuals younger than 25 years, individuals aged 25 to 65 years, and for individuals aged 65 years and older.

\section{Correction For Multiple Non-Independent Comparisons}

As mentioned above, we tested three different hypotheses: first, common pathway genes (BDNF and NTRK2) for the two drugs; second, serotonergic genes (HTR1A, HTR2A, $T P H 1, T P H 2$, and SLC6A4) for escitalopram; and third, noradrenergic genes (ADRA2A and SLC6A2) for nortriptyline, which resulted in a total of 123 tests across the three hypotheses.

Several approaches were applied to correct for multiple non-independent comparisons. First, the effective number of comparisons (Meff) was computed using the web-based SNPspd software (http://gump.qimr.edu.au/general/daleN/ SNPSpD/) with the method described by Li and Ji, (2005), (Nyholt, 2004) taking into account the linkage disequilibrium (LD) between markers. The threshold for significance was then calculated as $\alpha_{\text {corr }}=0.05 /$ Meff. Based on this method, the Meff for the 123 tests performed in the whole sample was found to be 81.25. The threshold for significance was therefore set to $0.05 / 81.25=6.15 \times 10^{-4}$. As specific hypotheses were tested, we also calculated the hypotheseswide Meffs: the Meffs for the tests examining the effect of common pathway genes on suicidality during treatment with both escitalopram and nortriptyline treatment, of serotonergic genes during esitalopram treatment, and of noradrenergic genes during nortriptyline treatment were found to be $31.089,34.161$, and 16 , respectively. The corresponding $p$-values for significance were therefore set to $0.0016,0.0015$, and 0.0031 , respectively.

Second, a false discovery rate was applied to quantify the uncertainty across the multiple hypotheses tested in the 123 single-marker tests. The false discovery rate $q$-value, which denotes the posterior probability of false negatives among multiple findings for each of these 123 non-independent tests was calculated using the procedure described by Benjamini and Hochberg, (1995) and applied in QVALUE software (http://genomics.princeton.edu/storeylab/qvalue/).

\section{Haplotype Analysis}

In a second set of analyses, we augmented the single SNP tests with multimarker haplotype tests if more than one SNP in a gene was associated with the trait with a $p$-value of 0.05 or less.

Linkage disequilibrium between polymorphisms in our data and haplotype blocks were estimated using the Haploview software 3.2 (http://www.broad.mit.edu/mpg/ haploview). For the haplotype estimations, we used the sliding window procedure implemented in PLINK. Polymorphisms that were used for haplotype analyses were firstly those primarily associated with the trait ( $p$-value of 0.05 or less), then those that had a borderline significant $p$-value within the same gene (between 0.06 and 0.05 ), and finally those that have previously been shown to be functional. A permutation procedure was used to estimate the significance of the best result (10000 permutations). This procedure will do 10000 permutations for all the tested haplotypes and gives an empirical $p$-value correcting for the fact that many tests were performed. This provides a less conservative correction for multiple testing than a Bonferroni correction and reduces the risk of type II errors. Haplotypes with frequencies lower than $1 \%$ were excluded.

\section{Power Calculation}

Power to detect associations was estimated using the Genetic Power Calculator (http://pngu.mgh.harvard.edu/ purcell/gpc/). Thus, we determined that the case-control sample had $98 \%$ power to detect a risk allele in a common pathway gene with $15 \%$ frequency and a dominant genotype relative risk of 2.0 at $\alpha$ of 0.05 . The power dropped to $79 \%$ for the $\alpha$ corrected for hypothesis-wide effective number of independent comparisons $\left(\alpha_{\text {corr }}=0.0016\right)$. The sample provided $94 \%$ power to detect an effect of a marker in a serotonin-related gene at $\alpha=0.05$, with power reducing to $53 \%$ for $\alpha_{\text {corr }}=0.0015$. The sample provided $90 \%$ power to detect an effect of a marker in the noradrenergic-related genes at $\alpha=0.05$ and $49 \%$ at $\alpha_{\text {corr }}=0.0031$.

\section{Population Stratification}

Recruitment was restricted to subjects of European ancestry for at least two generations. We also investigated the genetic structure of the sample using the program STRUCTURE (Pritchard et al, 2000). Thirty-five SNPs selected as being informative for population stratification in a European population (Seldin et al, 2006) were genotyped and analyzed using 10000 burn-ins and 10000 repetitions under an admixture model with correlated allele frequencies in STRUCTURE. This analysis found no clustering within the sample (data not shown). Stratification was further estimated using the genomic control approach in PLINK (Purcell et al, 2007). Based on the same set of ancestryinformative SNPs, the genomic inflation factor was estimated to be 0.83 , confirming the absence of significant stratification in our sample (Dadd et al, 2008).

\section{RESULTS}

\section{Sample Characteristics}

The data included 127 single nucleotide polymorphisms (SNPs), the 5-HTTLPR and the BDNF-1040(GT)n. 


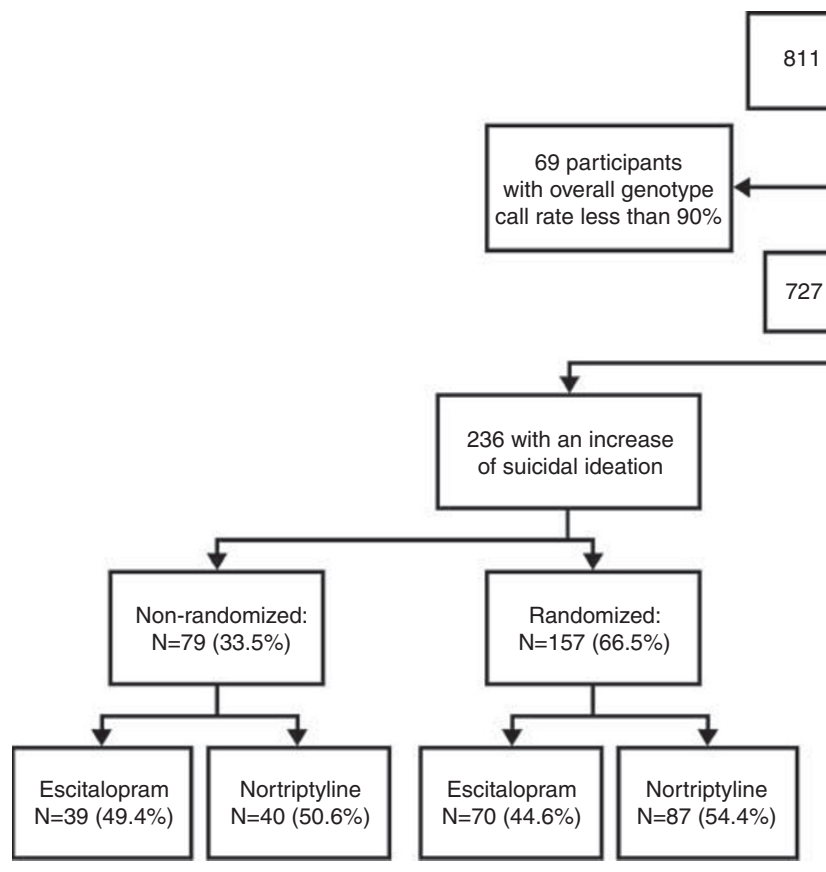

Figure I Flow of participants through the study.

Figure 1 displays the flow of participants throughout the study. Sixty-nine subjects (18 with and 51 without an increase in suicidal ideation) were excluded from the analyses owing to failing genotyping quality control (overall call rate less than 90\%). The genotyping rate for the remaining subjects was $97 \%$. Polymorphisms with departure from Hardy-Weinberg equilibrium at $p<0.001$ and/or a minor allele frequency of less than 0.05 (six polymorphisms) were also excluded. The final dataset consisted of 727 subjects (236 cases and 491 controls; 264 men and 463 women) and 123 polymorphisms. The mean age was 42.3 years (SD, 11.7). There were no significant differences between subjects excluded for genotyping quality control and those included in the analysis on clinical or demographic variables. There was no difference in gender and age between individuals with and without an increase in suicidal ideation (Table 1). Individuals with an increase in suicidal ideation had a higher baseline severity of depression (1.01 (0.58) vs $0.89(0.6), p=0.02$, as measured by the previously described 'observed mood' dimension, which does not include any items measuring suicidal ideation (Uher et al, 2008)), more depressive episodes (1.88 (0.73) vs $1.75(0.59), p=0.007)$ and not surprisingly a higher frequency of history of suicide attempts $(64(27.1 \%)$ vs 50 $(10.2 \%), p<0.0001)$ (Table 1). There was a significant difference in randomization, with individuals experiencing an increase in suicidal ideation being more often nonrandomly allocated than those without increase in suicidal ideation $(p=0.001)$ (Figure 1). Therefore, randomization status was used as a covariate in all analyses. Finally, as there were more dropouts among individuals with no increase in suicidal ideation and therefore a shorter period of risk, dropout status was also used as a covariate in the analyses.

During the 12 weeks of follow-up, individuals with an increase in suicidal ideation had a poorer outcome as measured by the 'observed mood' dimension $(b=0.12$;

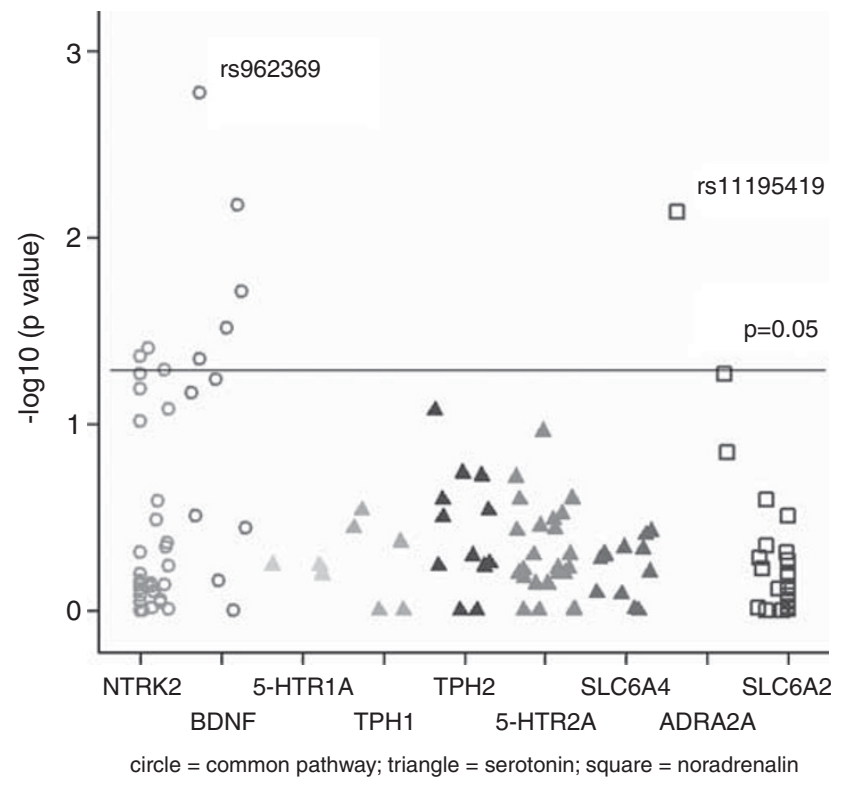

Figure 2 Association between increase in suicidal ideation on treatment and 123 polymorphisms in nine candidate genes: two common pathway genes (circles), five serotonergic genes (triangles) and two noradrenergic genes (squares).

$p=0.0001,95 \%$ confidence interval (CI) $0.06-0.18$ ). The change in the 'observed mood' dimension was therefore used as a covariate in a second sensitive analysis.

\section{Genetic Associations}

Common pathway genes. The candidate gene association results for an increase in suicidal ideation are shown in Figure 2. The strongest association within common pathway genes was found with the rs962369 marker in the gene 
Table 2 Association Results for Polymorphisms with p-values Less or Equal to 0.05

\begin{tabular}{|c|c|c|c|c|c|c|c|}
\hline Chromosome & SNP & Position (Mb) & Gene & Minor allele & Allelic odds ratio ${ }^{a}$ & $Z$ score & p-value \\
\hline 11 & rs962369 & 27690996 & BDNF & G & 1.504 & 3.183 & 0.001456 \\
\hline 11 & rs 11030102 & 27638172 & BDNF & G & 1.439 & 2.7 & 0.006925 \\
\hline 10 & rsll|95419 & | 12829358 & ADRA2A & A & 1.747 & 2.684 & 0.007274 \\
\hline 11 & rsllo30I0I & 27637320 & BDNF & $\mathrm{T}$ & 0.7652 & -2.372 & 0.01771 \\
\hline 11 & rs2030324 & $2768349 \mid$ & BDNF & C & 0.7916 & -2.089 & $0.0367 \mid$ \\
\hline 9 & rs3824519 & 84799558 & NTRK2 & $\mathrm{T}$ & 1.574 & 1.986 & 0.04709 \\
\hline
\end{tabular}

Age $<25$ years old, $N=52$ ( 14 cases and 38 controls)

None

Age $\geqslant 25$ and Age $<65$ years old, $N=658$ (214 case and 444 controls)

\begin{tabular}{|c|c|c|}
\hline rs962369 & 27690996 & BDNF \\
\hline $\mathrm{rs} / 1030102$ & 27638172 & BDNF \\
\hline rsl|03010। & 27637320 & BDNF \\
\hline rs 11195419 & | 12829358 & ADRA2A \\
\hline rs2030324 & $2768349 \mid$ & BDNF \\
\hline rs 12273363 & $2770 \mid 435$ & BDNF \\
\hline rs 10835210 & 27652486 & BDNF \\
\hline rs। I 87352 & 84523011 & NTRK2 \\
\hline rs3824519 & 84799558 & NTRK2 \\
\hline
\end{tabular}

BDNF

\begin{tabular}{ll}
$G$ & 1.612 \\
$G$ & 1.508 \\
$A$ & 0.7241 \\
$C$ & 1.815 \\
$C$ & 0.7447 \\
$A$ & 1.426 \\
$A$ & 0.765 \\
& 1.3 \\
\hline & 1.666
\end{tabular}

coding for BDNF with a $p$-value of 0.0015 (Table 2). This result was significant after correction for hypothesis-wide Meff. The two strongest associations (rs962369 and rs11030102) had a hypothesis-wide $q$-value of 0.06 and 0.14 , respectively, suggesting that these findings were unlikely to be false ones. The most significant polymorphism in the NTRK2 gene was rs1439050 with a $p$-value of 0.043 , which did not survive gene-wide nor hypothesis-wide Meff-based corrections.

The BDNF gene comprises a single block with strong LD between all polymorphisms (Figure 3 ). For the haplotype analyses of the $B D N F$ gene, we focused our attention on the five significant SNPs (rs962369, rs11030102, rs11030101, rs2030324, and rs12273363) and the two known functional polymorphisms: the Val66Met (rs6265) and the GT(n) repeat in the BDNF-LCPR (Egan et al, 2003; Okada et al, 2006). A sliding window procedure detected a GSG haplotype composed of the two known functional polymorphisms and rs962369 as associated with increase in suicidal ideation during treatment $(0.303$ vs 0.234 , $p=0.004$ ) (Table 3). This remained significant after 10000 permutations with a $p$-value of 0.04 . The strongest association within the NTRK2 gene was with a haplotype composed of the four most strongly associated SNPs: rs1439050, rs1187352, rs1778933, and rs3824519 located just before block 1, and within the blocks 1 and 4 (Figure 4). There was a higher frequency of the GGTT haplotype among individuals with an increase in suicidal ideation (0.044 vs $0.019 ; p=0.0052$ ) and a higher frequency of the GGTC haplotype among individuals with no increase in suicidal ideation during antidepressant treatment $(0.474$ vs 0.563 ; $p=0.0013$ ) (Table 3). These remained significant after 10000 permutations with $p$-values of 0.046 and 0.014 , respectively.

Two BNDF polymorphisms have been shown to be functional, the Val66Met (rs6265) and the GT(n) repeat in the BDNF-LCPR (Egan et al, 2003; Okada et al, 2006), both in the same LD block. We therefore focused on these two polymorphisms to test for a possible interaction with the NTRK2 gene. The effective number of comparisons for the 33 SNPs in the NTRK2 gene was calculated to be 22.07. The a priori $p$-value for significance for the interaction between the two polymorphisms in the BDNF gene and the SNPs within the NTRK2 gene was therefore set to $0.05 / 22.07 \times 2=0.0011$. Using this threshold, we found a significant interaction between the BDNF GT(n) repeat and SNPs in the $3^{\prime}$ region of NTRK2 (lowest $p$-value $=0.00026$ ) (Table 4$)$.

\section{Results By Drug}

No marker within the serotonergic candidate genes was associated with an increase in suicidal ideation in the escitalopram treated group (Figure 2). 


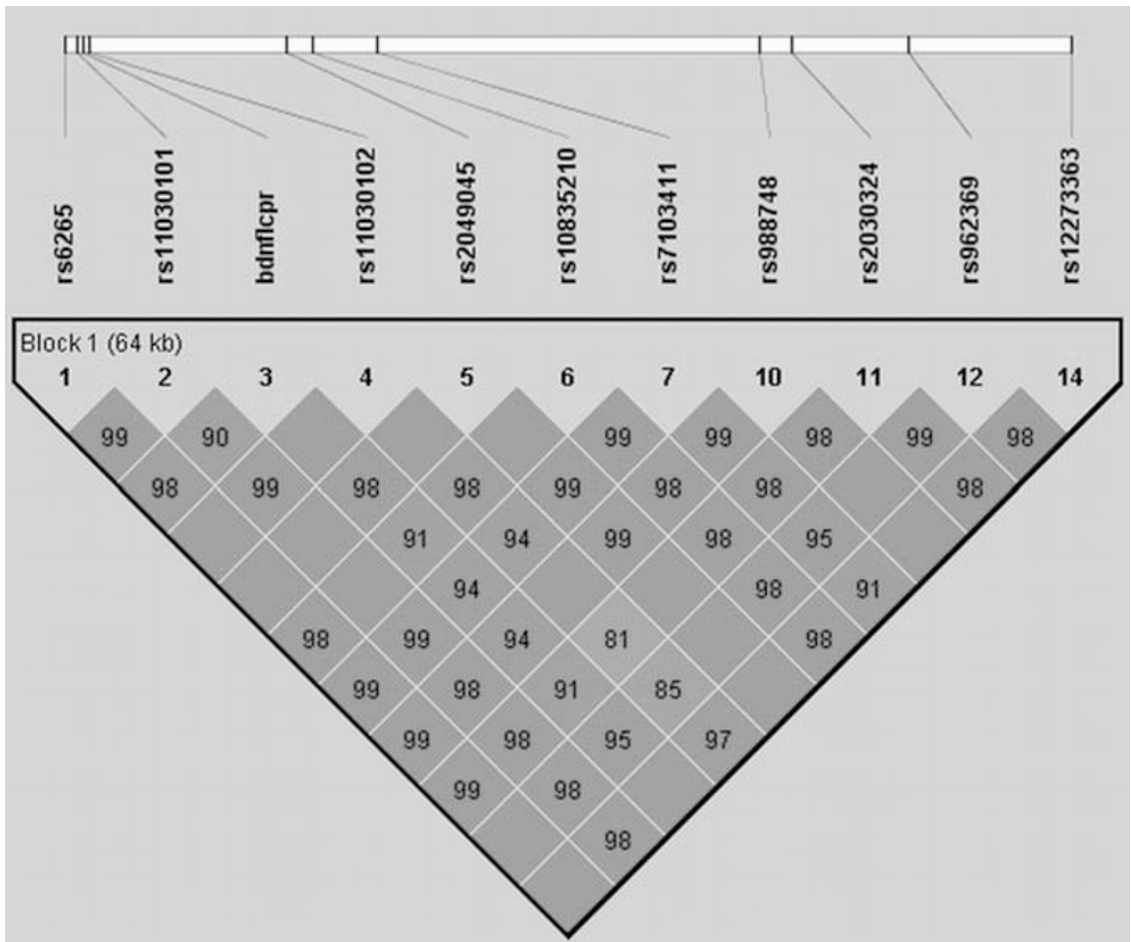

Figure 3 Linkage disequilibrium (as measured by $D^{\prime}$ ) between the II polymorphisms in BDNF.

Table 3 Haplotype Association Results for Three Polymorphisms in BDNF and Four SNPs in NTRK2

\begin{tabular}{|c|c|c|c|c|c|}
\hline Haplotype & $\begin{array}{l}\text { Frequency } \\
\text { in cases }\end{array}$ & $\begin{array}{l}\text { Frequency } \\
\text { in controls }\end{array}$ & $\angle R T^{\mathrm{c}}$ & p-value & $\begin{array}{c}\text { P after } 10000 \\
\text { permutations }\end{array}$ \\
\hline \multicolumn{6}{|l|}{$B D N F^{a}$} \\
\hline All & - & - & 10.52 & 0.01464 & NS \\
\hline GSG & 0.3034 & 0.2342 & 8.164 & 0.004272 & 0.04 \\
\hline GLA & 0.02835 & 0.0486 & 3.295 & $0.0695 \mid$ & NS \\
\hline GSA & $0.47 \mid I$ & 0.515 & 2.498 & 0.114 & NS \\
\hline ALA & 0.1971 & 0.2022 & 0.05301 & 0.8179 & NS \\
\hline \multicolumn{6}{|l|}{$N T R K 2^{b}$} \\
\hline All & - & - & 21.24 & 0.006527 & 0.051 \\
\hline GACT & $0.0157 \mid$ & 0.016 & 0.001744 & 0.9667 & NS \\
\hline TACT & 0.02256 & 0.01754 & 0.4329 & 0.5106 & NS \\
\hline GGTT & 0.04396 & 0.0188 & 7.811 & 0.005194 & 0.046 \\
\hline TGCC & 0.0189 & 0.02847 & 1.197 & 0.2739 & NS \\
\hline TATC & 0.05362 & 0.06003 & 0.2433 & 0.6219 & NS \\
\hline TGTC & 0.09769 & 0.07357 & 2.516 & 0.1127 & NS \\
\hline GACC & 0.09862 & 0.09286 & 0.1254 & 0.7233 & NS \\
\hline TACC & 0.175 & 0.1297 & $5.37 \mid$ & 0.02048 & NS \\
\hline GGTC & 0.474 & 0.5631 & $|0.3|$ & 0.001323 & 0.014 \\
\hline
\end{tabular}

aHaplotype: rs6265; BDNF (GT)n repeat; rs962369.

bHaplotype: rs 1439050; rs I 187352; rs I778933; rs3824519.

'LRT: likelihood ratio test.

For nortriptyline, rs11195419 in ADRA2A was significantly associated with an increase in suicidal ideation (the minor A allele increasing the risk with an OR of 1.75, $p=0.007$ ) (Table 2). This result was not significant after gene-wide or hypothesis-wide Meff-based correction for multiple comparisons. However, there was a significant drug by gene interaction for this SNP $(z=-5.26$; $p<0.0001)$. This interaction was explained by a higher proportion of an increase in suicidal ideation among individuals carrying the $\mathrm{A}$ allele and taking nortriptyline $(\mathrm{OR}=2.33 ; 95 \% \mathrm{CI}: 1.47-3.69 ; p=0.001$ ) (Figure 5). In addition, there was a gender by gene interaction in the group taking nortriptyline $(z=3.23 ; p=0.001)$. This interaction was explained by a higher proportion of males having an increase in suicidal ideation when carrying the $\mathrm{A}$ allele than if homozygous for the $\mathrm{C}$ allele; whereas, this observation was less pronounced in the female sample (Figure 5).

After adjustment for history of suicide attempts, baseline severity of depression and magnitude of changes in the 'observed mood' dimension factor in sensitivity analysis, all results remained unchanged and even became more significant $(p=0.00125$ for $\mathrm{rs} 962369 ; p=0.004486$ for rs11030102; $p=0.03568$ for $r$ 11030101; and $p=0.04679$ for rs12273363 in the $B D N F$ gene, $p=0.00156$ for rs1187352; $p=0.003295$ for $\mathrm{rs} 1778933 ; p=0.01556$ for $\mathrm{rs} 3824519$; and $p=0.04435$ for $\mathrm{rs} 1439050$ in the NTRK2 gene, and $p=0.005031$ for $\operatorname{rs} 11195419$ in the ADRA2A gene. The rs1187352 in the NTRK2 involved in the haplotype association described above reached significance after correction for hypothesis-wide Meff confirming the involvement of this gene in suicidal ideation.

Stratification by age revealed a strong association with increases in suicidal ideation for the large group of subjects aged 25 to 64 years ( $p=0.00055$ for rs962369) (Table 2$)$. The small sizes of the under 25 and above 65 age groups precluded meaningful tests of associations in these strata. 


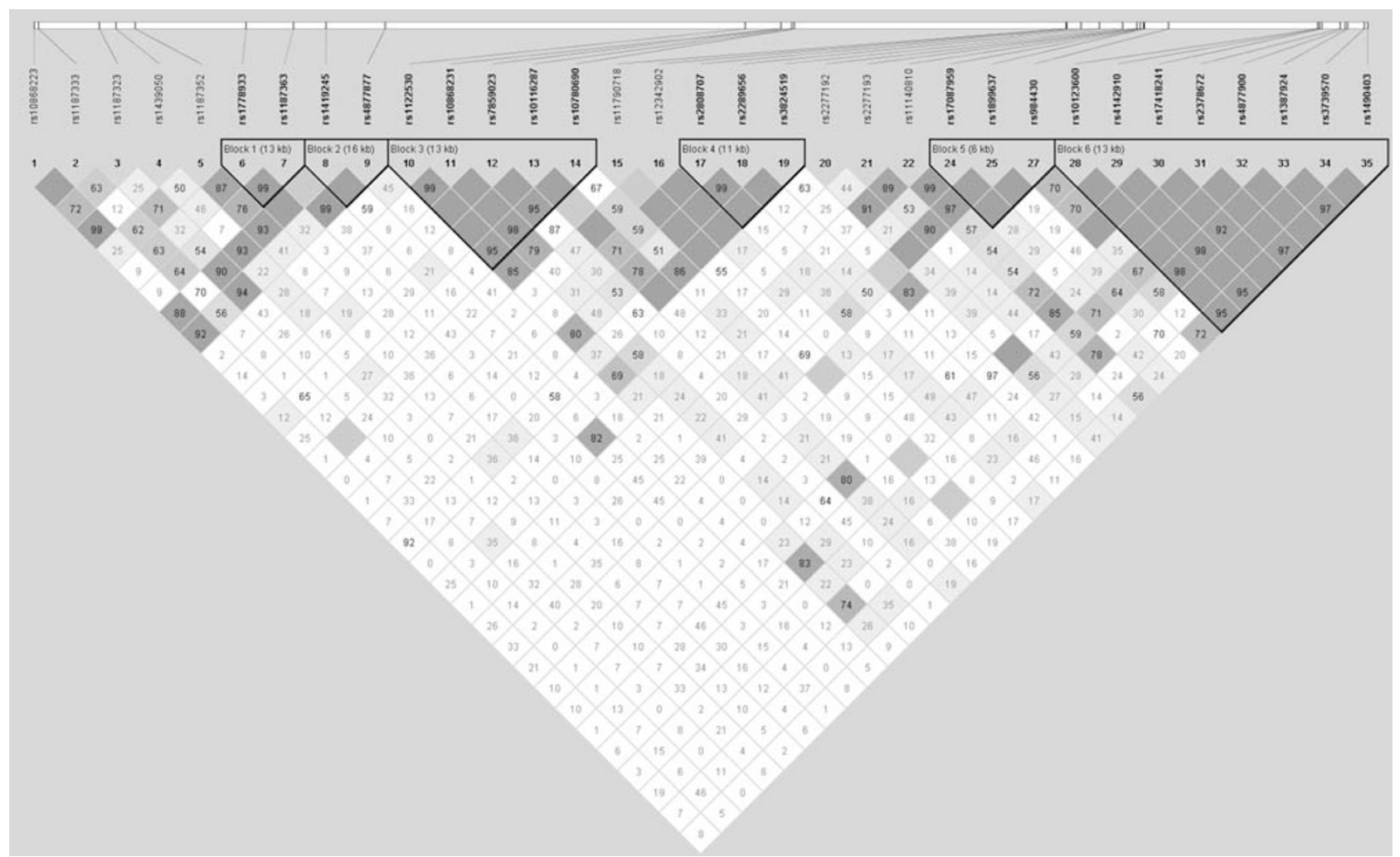

Figure 4 Linkage disequilibrium (as measured by $D^{\prime}$ ) in variants tested in NTRK2.

Table 4 Interactions Between NTRK2 SNPs (on chromosome 9) and the BDNF (GT)n Repeat (on chromosome I I). All Interactions Significant at the Nominal Level of $p \leqslant 0.05$ are Listed.

\begin{tabular}{ll}
\hline NTRK2 SNP & P for interaction with BDNF (GT)n repeat \\
\hline rs I I I 40810 & 0.01112 \\
rs 2378672 & 0.008645 \\
rs 4877900 & 0.00026 \\
rs 1387924 & 0.03377 \\
rs 1490403 & 0.01366 \\
\hline
\end{tabular}

\section{DISCUSSION}

We found that variants in the genes encoding BDNF and its receptor (NTRK2) were associated with an increase in suicidal ideation during antidepressant treatment in subjects suffering from major depressive disorder. Moreover, we showed an even stronger effect for an interaction between the known functional BDNF-LCPR GT(n) polymorphism and SNPs in the $3^{\prime}$ region of NTRK2. Finally, we found a drug by gene interaction involving $A D R A 2 A$, with a specific effect of rs11195419 in men taking nortriptyline.

BDNF has an important role not only in the regulation and growth of 5-HT neurons, but is also a mediator of the neural plasticity in response to acute and chronic stress (Berton et al, 2006; Murakami et al, 2005; Pizarro et al, 2004; Tsankova et al, 2006). As both the 5-HT system and childhood maltreatment have been associated with suicidal behavior, the BDNF gene is a strong candidate for involvement in suicidality. The involvement of BDNF in $\mathrm{SB}$ is supported by several studies showing a decreased level of BDNF in the hippocampus and prefrontal cortex (PFC) of suicide victims and in the cerebrospinal fluid (CSF) and plasma of suicide attempters (Dwivedi et al, 2003b; Karege et al, 2005; Kim et al, 2007). Although chronic antidepressant treatment has been shown to increase central expression of BDNF (Altar et al, 2003; Coppell et al, 2003), it has also been postulated that acute treatment could temporarily reduce BDNF transcripts (Dias et al, 2003). Decreased BDNF levels have been reported in the plasma and CSF of subjects with $\mathrm{SB}$, so that it could be postulated that SB would be most pronounced in the acute phase of treatment. In our study, however, the risk of an increase in total suicidal ideation (combining treatment-emergent and treatmentworsening) was approximately evenly distributed through the assessment period (Perroud et al, 2009). It is therefore possible that the reduction in BDNF level is more persistent in suicidal subjects than in non-suicidal ones. As BNDFdeficient mice display aggressive behaviors (Lyons et al, 1999), antidepressant-induced changes in BDNF could be associated with a higher tendency to impulsivity and aggression.

Among the two known functional polymorphisms of $B D N F$, the Val66Met (rs6265) was the most studied in SB. The $\mathrm{G}>\mathrm{A}$ substitution in nucleotide position 196 results in a valine ( $\mathrm{Val}$ ) to methionine (Met) change at amino acid position 66, with BDNF secretion having being shown to be reduced in 66Met BDNF neurons (Egan et al, 2003). To our knowledge, only four studies in populations of European 

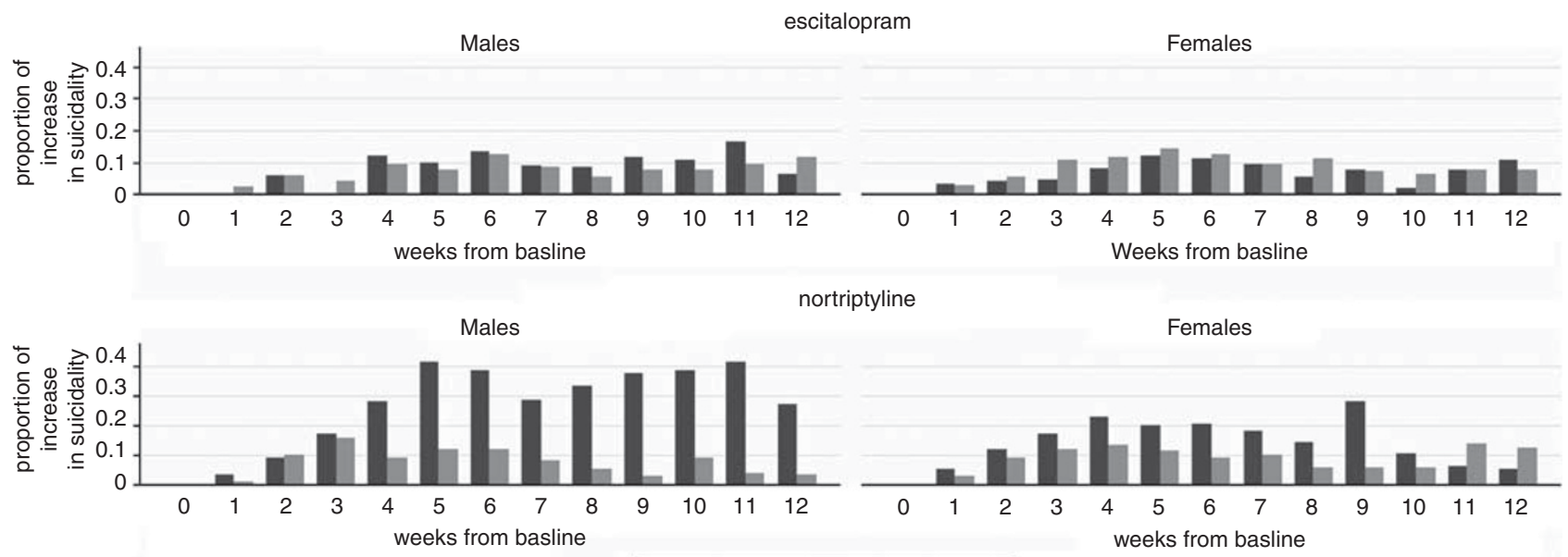

ortriptyline

$A A+A C$

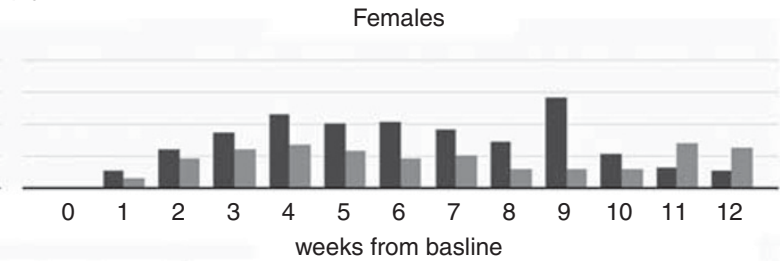

CC

Figure 5 Proportion of increase in suicidal ideation by carriers of the AA + AC genotypes vs CC genotypes of the rs I I I 954 I9 in ADRA2A stratified by drug and by gender during the 12 weeks of follow-up.

ancestry have investigated this polymorphism in relation to SB (Perroud et al, 2008; Sarchiapone et al, 2008; Vincze et al, 2008; Zarrilli et al, 2008). In two of these studies, the $\mathrm{Val}$ allele was found to be associated with increased risk of SB. This is consistent with our finding showing that individuals carrying the Val allele in the GSG haplotype were at higher risk of suicidal ideation during treatment. This is also consistent with a recent study showing that the Met allele appears to counteract the effect of the 5-HTTLPR $S$ allele on amygdala function (Pezawas et al, 2008) suggesting that the Val allele is associated with impaired cognitive function and increased risk of suicide. Our results are also supported by a recent finding implying that short alleles (equivalent to our S allele) of the GT(n) repeat in the BDNF-LCPR were associated with lower transcriptional activity compared with major alleles and were associated with bipolar disorder (Okada et al, 2006). Taken together, our findings therefore suggest that the $\mathrm{S}$ allele of the $B D N F$ GT(n) repeat in association with the Val allele of the Val66Met may confer susceptibility to suicidal ideation during antidepressant treatment by modifying the transcriptional activity of the $B D N F$ gene.

As with BDNF, NTRK2 mRNA has been shown to be significantly lower in the prefrontal cortex and hippocampus of suicide victims than in controls (Dwivedi et al, 2003b; Tsai, 2005). Our results suggest an involvement not only of BNDF, but also of the whole neurotrophic factor system in SB and are concordant with Tsai's hypothesis (Tsai, 2005), which postulates that the best candidates for treatment-emergent suicidal ideation are $B D N F$ and its receptor gene NTRK2.

Recently, Perlis et al (2007) found that treatmentemergent suicidal ideation was associated with SNPs within the cyclic adenosine monophosphate (cAMP) response element binding (CREB) protein gene. Interestingly, it has been hypothesized that activation of CREB increases BDNF transcription and that conversely, decreased levels of CREB found in the brain of suicide victims could be responsible for the decreased BDNF expression in these subjects (Dwivedi et al, 2003a; Finkbeiner, 2000). Our results are therefore concordant with the findings of Perlis et al (2007), supporting an involvement of this system in increasing suicidal ideation during antidepressant treatment.

In antidepressant-specific analyses, we found that the ADRA2A gene was associated with an increase in suicidal ideation in men taking nortriptyline. Suicide victims have increased numbers of alpha- ${ }_{2 \mathrm{~A}}$ adrenergic receptors and mRNA expression in the hippocampus and frontal cortex (Escriba et al, 2004; Garcia-Sevilla et al, 1999; Gonzalez et al, 1994). Moreover, the ADRA2A receptor gene is located on chromosome 10q24-q26, a region that has been linked to SB in genome-wide linkage studies (Cheng et al, 2006). The noradrenergic system is thought to be implicated in aggressive and impulsive behaviors, and enhanced noradrenergic activity may have a role in SB (Oquendo and Mann, 2000). Our findings of a significant association between increasing suicidal ideation during treatment and the ADRA2A gene implies that dysregulation of noradrenergic transmission through alpha-2A adrenergic receptors may have an impact on aggressive and impulsive behaviors, especially in men taking nortriptyline. Our results therefore support the hypothesis that hypersensitivity or enhanced activity of these receptors could, in part, explain why some individuals have an increase in suicidal ideation during treatment with a noradrenergic antidepressant (Perroud et al, 2009). Our results also suggest that this sensitivity is explained by a genetic polymorphism within the relevant gene $(A D R A 2 A)$. Indeed, carriers of the $\mathrm{A}$ allele of the rs11195419 were at higher risk of suicidal ideation on treatment when taking nortriptyline. Through the interactive web-based SNP analysis tool Pupasuite (http:// pupasuite.bioinfo.cipf.es/), we found that rs11195419 is located at an exonic splicing enhancer (ESE) and therefore may alter the normal splicing pattern. Moreover, based on data from the International HapMap project (http:// www.hapmap.org/), rs11195419 is located in a region of strong LD, including two previously investigated polymorphisms: N251K and C-129G. N251K has been shown to be functional (Small and Liggett, 2001) and has been reported to be involved in the susceptibility to suicide 
completion (Sequeira et al, 2004). The C-129G polymorphism has been associated with hostility, impulsivity, irritability, and suicide completion (Comings et al, 2000; Fukutake et al, 2008). Taken together, these data point to the involvement of the ADRA2A receptor in susceptibility to suicidality.

\section{Limitations}

The main limitation is the absence of a placebo control group, which means that we are not able to test whether the increase of SB is attributable or at least partially attributable to treatment with the antidepressants studied. Although this is one of the largest prospective pharmacogenetic studies in depression, we still do not have sufficient power to detect moderate associations at a $p$-value of 0.001 or less. We therefore applied a Meff correction. The result observed with $B D N F$ was significant after hypothesis-wide Meff-based correction. Moreover, the $q$-value for the three top associations (two polymorphisms, rs962369 and rs11030102, in $B D N F$ and the rs11195419 in ADRA2A) were given a value of 0.18 or less, indicating that these results were unlikely to be false. In other words, taking into account the number of comparisons performed, each of these results is more than four times more likely to be a true positive than a false positive.

We observed that individuals with an increase in suicidal ideation had less favorable response to treatment as calculated by the 'observed mood' dimension. This observation could potentially confound the genetic association results. To test the specificity of associations to suicidal ideation as opposed to general treatment response, we adjusted the analyses on the magnitude of change in the 'observed mood' dimension. This analysis confirmed that associated genetic markers were independent predictors of increase in suicidal ideation during treatment. Moreover, supporting this result, neither BDNF, NTRK2, nor ADRA2A genes were found to be predictors of overall response to antidepressants in the same sample (Uher et al, 2009a).

Although reports from the FDA (US Food and Drug Administration, 2006) suggested that treatment-related suicidal ideation was more common in individuals aged under 25 years, we did not have sufficient power to detect even a moderately strong genetic association in this age group.

The remaining question is whether a history of a previous suicide attempt is part of the phenotype of suicidal ideation during treatment. Even though the results did not change after adjustment for history of suicide attempt, as we found the same genes to be associated with suicidal ideation and suicide attempts in this data set, this strongly suggests a substantial overlap between these two phenotypes. Further research in this field is indicated.

\section{Conclusion}

We have found that BDNF and its receptor are associated with an increase in suicidal ideation in subjects taking either escitalopram or nortriptyline. Moreover, we have shown that noradrenergic antidepressants enhance suicidal ideation in men, with the most likely mechanism being through the ADRA2A. We suggest that future studies should include investigation of whether BDNF and norepinephrine receptors are involved in a more general susceptibility to suicidal behavior.

\section{ACKNOWLEDGEMENTS}

The GENDEP study was funded by a European Commission Framework 6 grant, EC Contract Ref.: LSHB-CT-2003503428. Nader Perroud was founded by the Swiss National Science foundation: PASMA-118605. Lundbeck provided both nortriptyline and escitalopram free of charge for the GENDEP study. GlaxoSmithKline contributed by funding an add-on project in the London center. In its latter stages, GENDEP received additional funding at the Institute of Psychiatry site from the Biomedical Research Center for Mental Health at the Institute of Psychiatry, King's College London and South London, and Maudsley NHS Foundation Trust (National Institute for Health Research, Department of Health, UK). However, the authors work was independent of the funders, with the legal sponsor of the study being the Institute of Psychiatry at King's College London. The funders had no role in the design and conduct of the study, in data collection, analysis, interpretation, or writing of this report.

We acknowledge the contribution of our co-workers and collaborators: Helen Dean, Kelly Halton, Cerisse Gunasinghe, Jacqui Kraska, Georgina Hosang, Desmond Campbell, Richard J Williamson, Julien Mendlewicz, Christine Schmäl, Susanne Höfels, Anna Schuhmacher, Ute Pfeiffer, Sandra Weber, Erik Roj Larsen, Anne Schinkel Stamp, Dejan Kozel, Alenka Tancic, Jerneja Sveticic, Zrnka Kovacic, Paweł Kapelski, Maria Skibiñska, Piotr M Czerski, Ophelia Beer, Nicola Reynolds, Linda Southwick, Julia Wood, Katharine Mead, Ylva Dahlin, Rachel Marsh, Sam Keating, Yohan Bhairo, Julian Childs, Zrnka Kovacic, Maja Bajs, Aleksandra Rajewska, Anna Leszczynska, and Caterina Giovannini. We particularly acknowledge the contribution of Professor Jorge Perez, who was the Lead Investigator at Brescia, Italy, and who passed away in October 2007, and to note the important contribution of our co-author, Dr Andrej Marusic, the Lead Investigator at Ljubljana, Slovenia, who passed away in June 2008.

\section{DISCLOSURE/CONFLICT OF INTEREST}

Perroud, Uher, Smith, Marusic, Hauser, Rietschel, Mors, Placentino, Jorgensen, Kapelski, Bonvicini, Zobel, Petrovic, Schulze, Gupta, Lewis, Huezo-Diaz, Gray, and Craig have no conflicting financial interests. Henigsberg, Kalember, and Souery have participated in clinical trials sponsored by pharmaceutical companies including GlaxoSmithKline and Lundbeck. Maier, Zobel, Farmer, McGuffin, and Aitchison have received consultancy fees and honoraria for participating in expert panels for pharmaceutical companies including Lundbeck and GlaxoSmithKline.

\section{REFERENCES}

Altar CA, Whitehead RE, Chen R, Wortwein G, Madsen TM (2003). Effects of electroconvulsive seizures and antidepressant drugs on 
brain-derived neurotrophic factor protein in rat brain. Biol psychiatry 54: 703-709.

Bellivier F, Chaste P, Malafosse A (2004). Association between the TPH gene A218C polymorphism and suicidal behavior: a metaanalysis. Am J Med Genet B Neuropsychiatr Genet 124B: 87-91.

Benjamini Y, Hochberg Y (1995). Controlling the false discovery rate: a practical and powerful approach to multiple testing. $J R$ Stat Soc, Series B 57: 289-300.

Berton O, McClung CA, Dileone RJ, Krishnan V, Renthal W, Russo $\mathrm{SJ}$ et al (2006). Essential role of BDNF in the mesolimbic dopamine pathway in social defeat stress. Science (New York, NY) 311: 864-868.

Brent DA, Mann JJ (2005). Family genetic studies, suicide, and suicidal behavior. Am J Med Genet C Semin Med Genet 133C: 13-24.

Cheng R, Juo SH, Loth JE, Nee J, Iossifov I, Blumenthal R et al (2006). Genome-wide linkage scan in a large bipolar disorder sample from the National Institute of Mental Health genetics initiative suggests putative loci for bipolar disorder, psychosis, suicide, and panic disorder. Mol psychiatry 11: 252-260.

Cipriani A, Furukawa TA, Salanti G, Geddes JR, Higgins JP, Churchill $\mathrm{R}$ et al (2009). Comparative efficacy and acceptability of 12 new-generation antidepressants: a multiple-treatments meta-analysis. Lancet 373: 746-758.

Comings DE, Johnson JP, Gonzalez NS, Huss M, Saucier G, McGue $\mathrm{M}$ et al (2000). Association between the adrenergic alpha $2 \mathrm{~A}$ receptor gene (ADRA2A) and measures of irritability, hostility, impulsivity and memory in normal subjects. Psychiatric genet 10: $39-42$.

Coppell AL, Pei Q, Zetterstrom TS (2003). Bi-phasic change in BDNF gene expression following antidepressant drug treatment. Neuropharmacology 44: 903-910.

Courtet P, Jollant F, Castelnau D, Buresi C, Malafosse A (2005). Suicidal behavior: relationship between phenotype and serotonergic genotype. Am J Med Genet C Semin Med Genet 133C: 25-33.

Dadd T, Weale ME, Lewis CM (2008). A critical evaluation of genomic control methods for genetic association studies. Genet Epidemiol 33: 290-298.

de Bakker PI, Yelensky R, Pe'er I, Gabriel SB, Daly MJ, Altshuler D (2005). Efficiency and power in genetic association studies. Nat genet 37: 1217-1223.

Dias BG, Banerjee SB, Duman RS, Vaidya VA (2003). Differential regulation of brain derived neurotrophic factor transcripts by antidepressant treatments in the adult rat brain. Neuropharmacology 45: 553-563.

Dwivedi Y, Rao JS, Rizavi HS, Kotowski J, Conley RR, Roberts RC et al (2003a). Abnormal expression and functional characteristics of cyclic adenosine monophosphate response element binding protein in postmortem brain of suicide subjects. Arch gen psychiatry 60: 273-282.

Dwivedi Y, Rizavi HS, Conley RR, Roberts RC, Tamminga CA, Pandey GN (2003b). Altered gene expression of brain-derived neurotrophic factor and receptor tyrosine kinase $B$ in postmortem brain of suicide subjects. Arch gen psychiatry 60: 804-815.

Egan MF, Kojima M, Callicott JH, Goldberg TE, Kolachana BS, Bertolino A et al (2003). The BDNF val66met polymorphism affects activity-dependent secretion of BDNF and human memory and hippocampal function. Cell 112: 257-269.

Escriba PV, Ozaita A, Garcia-Sevilla JA (2004). Increased mRNA expression of alpha2A-adrenoceptors, serotonin receptors and mu-opioid receptors in the brains of suicide victims. Neuropsychopharmacology 29: 1512-1521.

Finkbeiner S (2000). Calcium regulation of the brain-derived neurotrophic factor gene. Cell Mol Life Sci 57: 394-401.

Freeman B, Smith N, Curtis C, Huckett L, Mill J, Craig IW (2003). DNA from buccal swabs recruited by mail: evaluation of storage effects on long-term stability and suitability for multiplex polymerase chain reaction genotyping. Behav genet 33: $67-72$.

Fukutake M, Hishimoto A, Nishiguchi N, Nushida H, Ueno Y, Shirakawa $\mathrm{O}$ et al (2008). Association of alpha(2A)-adrenergic receptor gene polymorphism with susceptibility to suicide in Japanese females. Prog Neuropsychopharmacol Biol Psychiatry 32: 1428-1433.

Garcia-Sevilla JA, Escriba PV, Ozaita A, La Harpe R, Walzer C, Eytan A et al (1999). Up-regulation of immunolabeled alpha2Aadrenoceptors, Gi coupling proteins, and regulatory receptor kinases in the prefrontal cortex of depressed suicides. J neurochem 72: 282-291.

Gonzalez AM, Pascual J, Meana JJ, Barturen F, del Arco C, Pazos A et al (1994). Autoradiographic demonstration of increased alpha 2 -adrenoceptor agonist-binding sites in the hippocampus and frontal cortex of depressed suicide victims. J neurochem 63: 256-265.

Huezo-Diaz P, Uher R, Smith R, Rietschel M, Henigsberg N, Marusic A et al (2008). Moderation of antidepressant response by the serotonin transporter gene in the GENDEP study. $\mathrm{Br} J$ Psychiatry 195: 30-38.

Karege F, Vaudan G, Schwald M, Perroud N, La Harpe R (2005). Neurotrophin levels in postmortem brains of suicide victims and the effects of antemortem diagnosis and psychotropic drugs. Brain Res Mol Brain Res 136: 29-37.

Kim YK, Lee HP, Won SD, Park EY, Lee HY, Lee BH et al (2007). Low plasma BDNF is associated with suicidal behavior in major depression. Prog Neuropsychopharmacol Biol Psychiatry 31: 78-85.

Laje G, Paddock S, Manji H, Rush AJ, Wilson AF, Charney D et al (2007). Genetic markers of suicidal ideation emerging during citalopram treatment of major depression. Am J Psychiatry 164: $1530-1538$.

Li D, He L (2007). Meta-analysis supports association between serotonin transporter (5-HTT) and suicidal behavior. Mol Psychiatry 12: 47-54.

Li J, Ji L (2005). Adjusting multiple testing in multilocus analyses using the eigenvalues of a correlation matrix. Heredity 95: 221-227.

Lyons WE, Mamounas LA, Ricaurte GA, Coppola V, Reid SW, Bora $\mathrm{SH}$ et al (1999). Brain-derived neurotrophic factor-deficient mice develop aggressiveness and hyperphagia in conjunction with brain serotonergic abnormalities. Proc Natl Acad Sci USA 96: $15239-15244$.

Mann JJ (2003). Neurobiology of suicidal behaviour. Nat Rev Neurosci 4: 819-828.

McGuffin P, Marusic A, Farmer A (2001). What can psychiatric genetics offer suicidology? Crisis 22: 61-65.

Murakami S, Imbe H, Morikawa Y, Kubo C, Senba E (2005). Chronic stress, as well as acute stress, reduces BDNF mRNA expression in the rat hippocampus but less robustly. Neurosci res 53: $129-139$.

Nyholt DR (2004). A simple correction for multiple testing for single-nucleotide polymorphisms in linkage disequilibrium with each other. Am J Hum Genet 74: 765-769.

Okada T, Hashimoto R, Numakawa T, Iijima Y, Kosuga A, Tatsumi $M$ et al (2006). A complex polymorphic region in the brainderived neurotrophic factor (BDNF) gene confers susceptibility to bipolar disorder and affects transcriptional activity. $\mathrm{Mol}$ psychiatry 11: 695-703.

Oquendo MA, Mann JJ (2000). The biology of impulsivity and suicidality. Psychiatr clin North Am 23: 11-25.

Perlis RH, Purcell S, Fava M, Fagerness J, Rush AJ, Trivedi MH et al (2007). Association between treatment-emergent suicidal ideation with citalopram and polymorphisms near cyclic adenosine monophosphate response element binding protein in the STAR ${ }^{\star} \mathrm{D}$ study. Arch gen psychiatry 64: 689-697. 
Perroud N, Courtet P, Vincze I, Jaussent I, Jollant F, Bellivier F et al (2008). Interaction between BDNF Val66Met and childhood trauma on adult's violent suicide attempt. Genes brain behav 7: 314-322.

Perroud N, Uher R, Marusic A, Rietschel M, Mors O, Henigsberg N et al (2009). Suicidality during treatment of depression with escitalopram and nortriptyline in GENDEP: A clinical trial. $B M C$ Psychiatry (in press).

Pezawas L, Meyer-Lindenberg A, Goldman AL, Verchinski BA, Chen G, Kolachana BS et al (2008). MET BDNF protects against morphological S allele effects of 5-HTTLPR. Mol psychiatry 13: 654.

Pizarro JM, Lumley LA, Medina W, Robison CL, Chang WE, Alagappan A et al (2004). Acute social defeat reduces neurotrophin expression in brain cortical and subcortical areas in mice. Brain research 1025: 10-20.

Pritchard JK, Stephens M, Donnelly P (2000). Inference of population structure using multilocus genotype data. Genetics 155: 945-959.

Purcell S, Neale B, Todd-Brown K, Thomas L, Ferreira MA, Bender $\mathrm{D}$ et al (2007). PLINK: A tool set for whole-genome association and population-based linkage analyses. Am J Hum Genet 81: 559-575.

Rabe-Hesketh S, Skrondal A (2005). Multilevel and longitudinal modeling using Stata. Stata Press: College Station, Texas.

Sanchez C, Bergqvist PB, Brennum LT, Gupta S, Hogg S, Larsen A et al (2003). Escitalopram, the S-(+)-enantiomer of citalopram, is a selective serotonin reuptake inhibitor with potent effects in animal models predictive of antidepressant and anxiolytic activities. Psychopharmacology (Berlin) 167: 353-362.

Sanchez C, Hyttel J (1999). Comparison of the effects of antidepressants and their metabolites on reuptake of biogenic amines and on receptor binding. Cell Mol Neurobiol 19: 467-489.

Sarchiapone M, Carli V, Roy A, Iacoviello L, Cuomo C, Latella MC et al (2008). Association of polymorphism (Val66Met) of brainderived neurotrophic factor with suicide attempts in depressed patients. Neuropsychobiology 57: 139-145.
Seldin MF, Shigeta R, Villoslada P, Selmi C, Tuomilehto J, Silva G et al (2006). European population substructure: clustering of northern and southern populations. PLoS Genet 2: e143.

Sequeira A, Mamdani F, Lalovic A, Anguelova M, Lesage A, Seguin $\mathrm{M}$ et al (2004). Alpha 2A adrenergic receptor gene and suicide. Psychiatry Res 125: 87-93.

Small KM, Liggett SB (2001). Identification and functional characterization of alpha(2)-adrenoceptor polymorphisms. Trends Pharmacol Sci 22: 471-477.

Tsai SJ (2005). Possible involvement of the BDNF-dependent pathway in treatment-emergent suicidality or decreased response to antidepressants. Med hypotheses 65: 942-946.

Tsankova NM, Berton O, Renthal W, Kumar A, Neve RL, Nestler EJ (2006). Sustained hippocampal chromatin regulation in a mouse model of depression and antidepressant action. Nat neurosci 9: 519-525.

Uher R, Farmer A, Maier W, Rietschel M, Hauser J, Marusic A et al (2008). Measuring depression: comparison and integration of three scales in the GENDEP study. Psychol Med 38: 289-300.

Uher R, Huezo-Diaz P, Perroud N, Smith R, Rietschel M, Mors O et al (2009a). Genetic predictors of response to antidepressants in the GENDEP project. Pharmacogenomics $J$ (in press).

Uher R, Maier W, Hauser J, Marusic A, Schmael C, Mors O et al (2009b). Differential efficacy of escitalopram and nortriptyline on dimensional measures of depression. Br J Psychiatry 194: 252-259.

US Food and Drug Administration (2006). Clinical review: relationship between antidepressant drugs and suicidality in adults. Available from: http://www.fda.gov/.

Vincze I, Perroud N, Buresi C, Baud P, Bellivier F, Etain B et al (2008). Association between brain-derived neurotrophic factor gene and severe form of bipolar disorder, but no interaction with the serotonin transporter gene. Bipolar Disord 10: 580-587.

Zarrilli F, Angiolillo A, Castaldo G, Chiariotti L, Keller S, Sacchetti $\mathrm{S}$ et al (2008). Brain derived neurotrophic factor (BDNF) genetic polymorphism (Val66Met) in suicide: a study of 512 cases. Am J Med Genet B Neuropsychiatr Genet 150B: 599-600. 